\title{
LA:UR- $98-3639$
}

Approved for public release; distribution is unlimited.

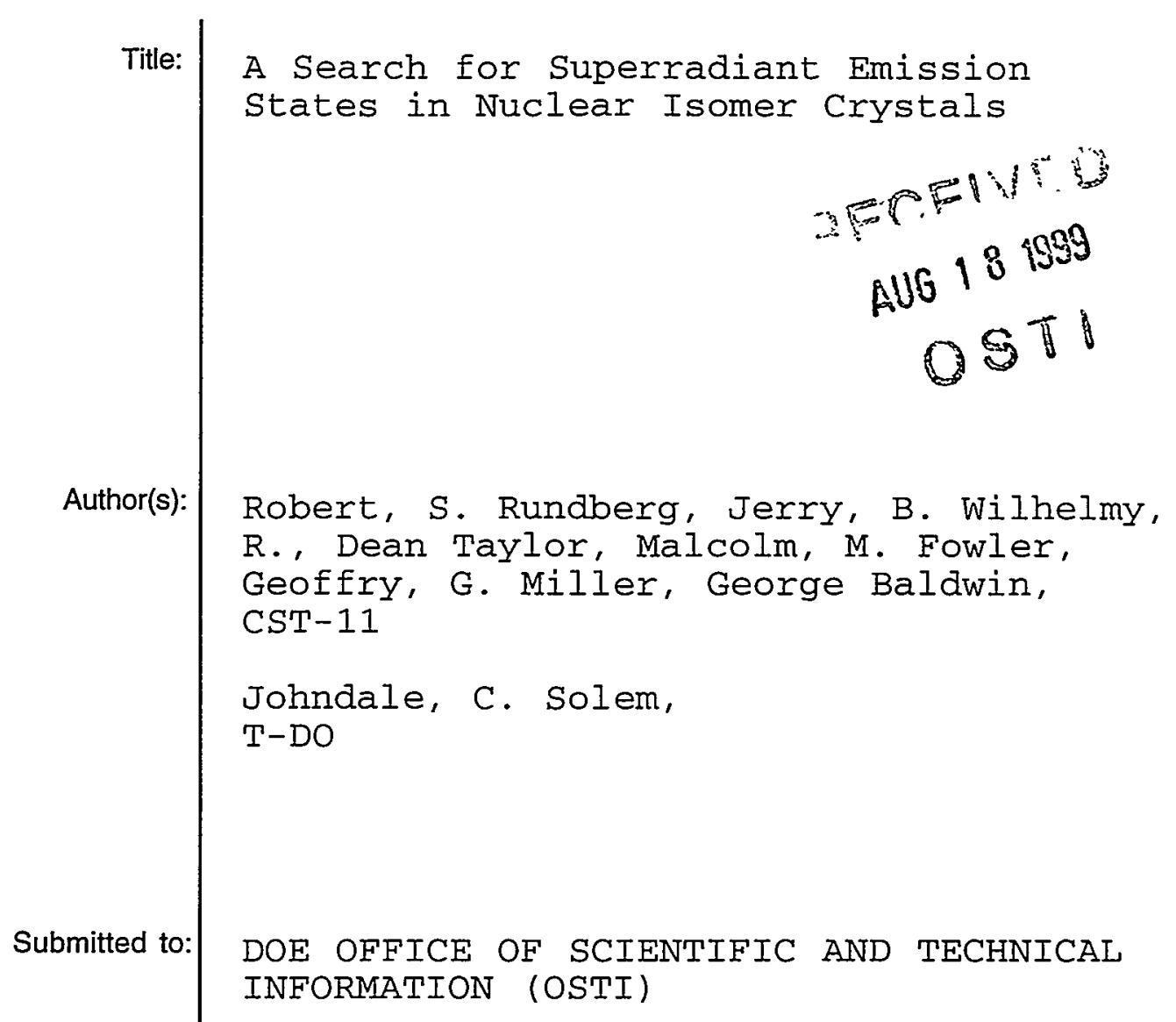

\section{Los Alamos}

NATIONALLABORATORY

Los Alamos National Laboratory, an affirmative action/equal opportunity employer, is operated by the University of California for the

U.S. Department of Energy under contract W-7405-ENG-36. By acceptance of this article, the publisher recognizes that the U.S.

Government retains a nonexclusive, royalty-free license to publish or reproduce the published form of this contribution, or to allow

others to do so, for U.S. Government purposes. Los Alamos National Laboratory requests that the publisher identify this article

as work performed under the auspices of the U.S. Department of Energy. The Los Alamos National Laboratory strongly supports

academic Ireedom and a researcher's right to publish; as an institution, however, the Laboratory does not endorse the viewpoint

of a publication or guarantee its technical correctness. 


\section{DISCLAIMER}

This report was prepared as an account of work sponsored by an agency of the United States Government. Neither the United States Government nor any agency thereof, nor any of their employees, make any warranty, express or implied, or assumes any legal liability or responsibility for the accuracy, completeness, or usefulness of any information, apparatus, product, or process disclosed, or represents that its use would not infringe privately owned rights. Reference herein to any specific commercial product, process, or service by trade name, trademark, manufacturer, or otherwise does not necessarily constitute or imply its endorsement, recommendation, or favoring by the United States Government or any agency thereof. The views and opinions of authors expressed herein do not necessarily state or reflect those of the United States Government or any agency thereof. 


\section{DISCLAIMER}

Portions of this document may be illegible in electronic image products. Images are produced from the best available original document. 


\title{
A Search for Superradiant Emission States in Nuclear Isomer Crystals
}

\author{
*Rundberg, Robert, S. \\ Wilhelmy, Jerry, B. \\ Taylor, R., Dean \\ Solem, Johndale, C. \\ Fowler, Malcolm, M. \\ Miller, Geoffry, G. \\ Baldwin, George
}

\begin{abstract}
This is the final report of a one-year, Laboratory Directed Research and Development (LDRD) project at Los Alamos National Laboratory (LANL). Our objective was to verify the stimulated emission of gamma rays from ${ }^{125 m} \mathrm{Te}$, as claimed by Russian scientists. The reported cross section for stimulated emission was sufficiently large to allow gain in a single-pass gamma-ray laser. The stimulated emission of gamma rays from a nuclear isomer is expected to result in collinear photons and, therefore, should be observable as a sum peak in the gamma-ray spectrum. Skorobogatov and Dzevitskii reported an increase of an order of magnitude in the sum peak $(218.56 \mathrm{keV})$ when a sample of beryllium telluride containing ${ }^{125 \mathrm{~m} T e}$ was cooled from room temperature to near-liquid-helium temperatures. We have repeated their experiment and have observed no increase in the sum peak above accidental summing. The upper limit for the stimulated-emission cross section based on the three-standard-deviation statistical error is $6.8 \times$ $10^{-21} \mathrm{~cm}^{2}$. This result is one order of magnitude lower than the cross section reported by Skorobogatov and Dzevitskii. Our cross section would not allow gain in a single-pass gamma-ray laser. Our results support the position of Baldwin and Solem rather than that of Kamenov.
\end{abstract}

\section{Background and Research Objectives}

Since shortly after the discovery of the Mössbauer effect [2] the possibility of creating a source of coherent gamma rays from nuclear isomers has been acknowledged $[3,4,5]$. Several reviews have been published on the theoretical basis for a gamma ray laser [6,7]. Several groups have examined the single excitation limit of superradiant states using synchrotron light sources to excite coherent states in single crystals containing ${ }^{57} \mathrm{Fe}$ $[8,9]$. These studies have verified that the photon channel can be significantly broadened in single crystals of nuclear isomers. The most recent of these experiments has even demonstrated that the coherent state can be turned on and off through application of strong magnetic fields [10]. But the technology for constructing a gamma ray laser has yet to be developed.

*Principal Investigator, e-mail: rundberg@lanl.gov 
This attempt to measure the cross section for stimulated emission in ${ }^{125 \mathrm{~m}} \mathrm{Te}$ was prompted by the observations reported by Skorobogatov and Dzevitskii [1]. They reported a cross section for the stimulated emission of $109.28 \mathrm{keV}$ from the nuclear isomer, ${ }^{125 \mathrm{~m}} \mathrm{Te}$, the magnitude of which would allow the construction of a gamma ray laser. The evidence for stimulated emission was based on a relatively simple experiment. The gamma ray spectrum from a polycrystalline rod of beryllium telluride containing ${ }^{125 \mathrm{~m}} \mathrm{Te}$ was measured at room temperature and at a temperature near the boiling point of liquid helium. A dramatic increase in the count rate at the energy corresponding to the sum of two 109.28 $\mathrm{keV}$ gamma rays ( $218.56 \mathrm{keV}$ ) was observed when the sample was cooled to liquid helium temperature. This result was consistent with the change in the fraction of recoilless transitions for a Mössbauer solid having a Debye temperature of about 400 degrees Kelvin. The Mössbauer effect is a necessary requirement for either resonant absorption or stimulated emission.

The exponential gain factor for a one-pass laser is

$$
K=f \sigma_{s t}[25 \mathrm{~m} T e]-\mu
$$

where ${ }^{\dagger}$ is the Mössbauer recoilless fraction, $\sigma_{\text {st }}$ the cross section for stimulated gamma ray emission, $\left[{ }^{[25 \mathrm{~m}} \mathrm{Te}\right]$ the concentration of ${ }^{125 \mathrm{~m}} \mathrm{Te}$ atoms per cubic centimeter, and $u$ the linear attenuation coefficient for 109.28-keV gamma rays in the medium. Applying the cross section reported by Skorobogatov and Dzevitskii [1], $7 \times 10^{-20} \mathrm{~cm}^{2}$, a Mössbauer recoilless fraction of .1, a linear attenuation coefficient of $4.8 \mathrm{~cm}^{-1}$, a density for BeTe of $3.6 \mathrm{~g} / \mathrm{cm}^{3}$, and an isomer population of 22.4 percent (this population could be achieved by milking tellurium from a ${ }^{125} \mathrm{Sb}$ generator), the gain $\mathrm{K}$ would be $21.1 \mathrm{~cm}^{-1}$. Thus the reported cross section would allow the construction of a $109-\mathrm{keV}$ gamma ray laser.

Two conflicting views of the theory of stimulated emission from three-level nuclear isomers have been published. Kamenov [11] published a treatment of stimulated emission that deviated from the original of Einstein [12] in that the decay of the first excited state competes with the resonant absorption of a gamma ray. Inherent in Kamenov's treatment is the assumption that the rate of excitation of the first excited state is a constant (Appendix A will derive the relations including temperature dependence of the excitation to the first excited state along with stimulated emission). This leads to cross-sections for stimulated emission and resonant absorption that are proportional to the sum of the level widths of the first and second excited states. The other view by Baldwin and Solem [13] utilizes 
Einstein's relations to demonstrate that the cross section (integral of a single level) for stimulated emission in a three-level system (where the first excited state is short lived) is extremely small.

The stimulated emission of photons is expected to be correlated in time and direction. Furthermore the line width of long-lived nuclear isomers is so narrow that the Mössbauer effect is needed to be resonant. Therefore it is not expected that stimulated emission will take place at room temperature, at least not for a 109.28-keV gamma ray, because the recoil energy is much larger than the line width. The experiment to measure stimulated emission of gamma rays is to look for photons that are correlated in time and direction, i.e., summed gamma rays, that appear at cryogenic temperatures but not at room temperature. In addition because the sample is rod shaped a greater effect should be expected along the longitudinal axis of the rod than perpendicular to the rod.

\section{Importance to LANL's Science and Technology Base and National R\&D Needs}

The ultimate goal of this research is to develop nuclear materials that could be used to design or be fabricated into a gamma ray laser. The applicable core competencies of LANL are Nuclear and Advanced Materials, Nuclear Science, Plasmas and Beams, and Nuclear Weapons Science and Technology.

\section{Scientific Approach and Accomplishments}

Beryllium telluride was synthesized by the procedure described in Skorobogatov and Dzevitskii [1]. SUPRASIL $®$ quartz capillary tubes (1.2-mm diameter) were internally coated with graphite by pyrolyzing natural gas, 21.2 milligrams of ${ }^{124} \mathrm{Te}$ (92 percent enrichment) was combined with 1.43 milligrams of powdered beryllium in an inert atmosphere, and the mixture transferred to the quartz capillary and sealed under vacuum.

The sample was heated in a programmable furnace to a temperature of 1100 degrees Celsius and soaked for one hour. The cooling ramp rate was 2 degrees per minute to encourage crystallization. The sample was then irradiated in the High Flux Beam Reactor, HFBR, at Brookhaven National Laboratory for 28 days in a thermal neutron flux of $1.4 \mathrm{x}$ $10^{14} \mathrm{n} / \mathrm{cm}^{2} \mathrm{~s}$. The thermal neutron fluence was $3.5 \times 10^{20} \mathrm{n} / \mathrm{cm}^{2}$. The conversion from ${ }^{124} \mathrm{Te}$ to ${ }^{125 \mathrm{~m}} \mathrm{Te}$ was approximately 0.02 percent.

After the first series of measurements the sample was reworked. The beryllium telluride was mixed with excess beryllium ( 5 milligrams) and annealed at 1100 degrees 
Celsius. This was done to ensure that the Debye temperature of the sample was high (about 400 degrees Kelvin).

The sample was cooled to liquid helium temperatures in a Janus Research liquid helium cryostat. A sketch of the experimental setup is shown in Figure 1. The detector was a Princeton Gamma Tech intrinsic germanium coaxial detector. A Tennelec 245 spectroscopy amplifier was used with some runs employing the linear pile up rejection feature. Pile up rejection was accomplished by comparing the peak amplitudes of the unipolar and bipolar signals. If the difference exceeded the unipolar channel noise level the event was rejected. This method eliminates ballistic deficit and charge trapping as well. The full width half maximum at $109.28 \mathrm{keV}$ was less than $1 \mathrm{keV}$ with an 8 microsecond shaping time and no pile up rejection. The data was acquired by a Canberra 35Plus multichannel analyzer.

Great care was taken to determine the accidental coincidence rate. The accidental coincidence rate depends on the pulse-pair resolving time of the overall spectrometer system. The rate of accidental coincidence, $R$, is

$$
R=\tau S^{2}
$$

where $S$ is the singles count rate and $\tau$ is pulse pair resolving time. The pulse pair resolving time is a constant that depends inter alia on the rise time and shape of pulses from the detector preamplifier and the shaping constant of the spectroscopy amplifier. The accidental coincidence rate is sensitive to the singles rate because of the quadratic dependence and is therefore sensitive to the reproducibility of the counting geometry, the decay rate, etc. Therefore most of the data will be represented as the ratio of the summing rate, $218.56 \mathrm{keV}$, to the singles rate, $109.28 \mathrm{keV}$, squared. Significant changes in the ratio as a function of sample temperature, source detector distance, or angle will be interpreted as evidence for stimulated emission. 

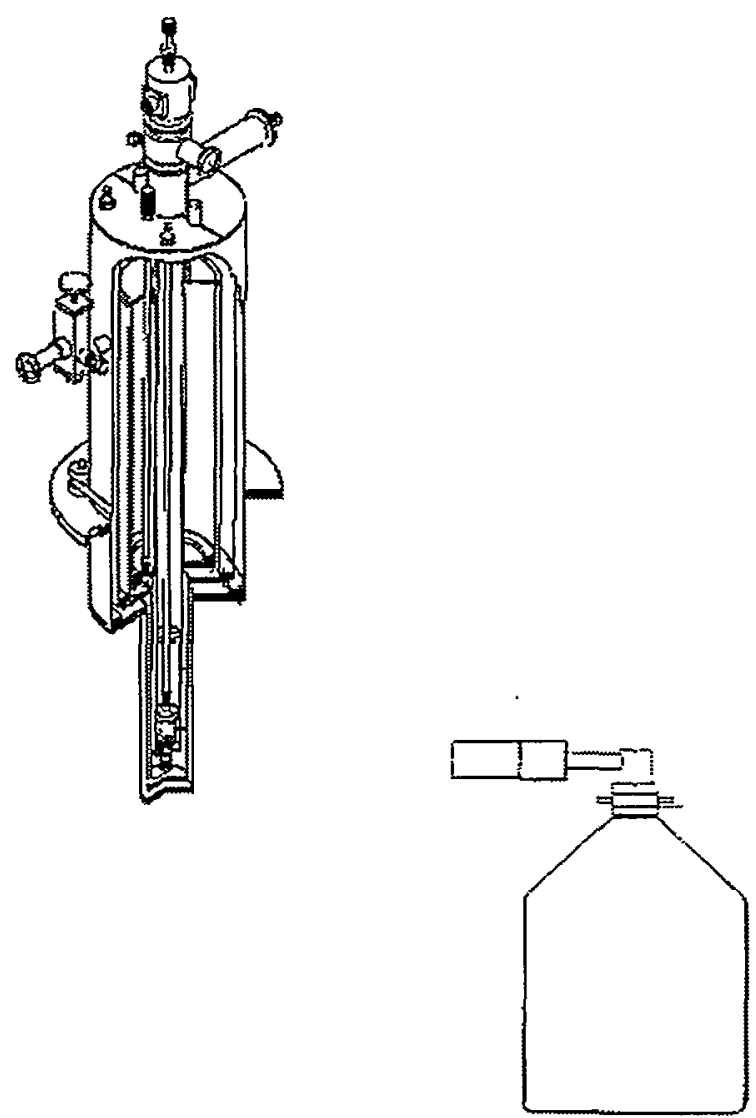

Figure 1. Experimental setup.

The Debye temperature of the reworked sample was determined by Mössbauer spectroscopy. The absorption of the $35.49-\mathrm{keV}$ gamma ray from the 1.48 nanosecond first excited state was measured as a function of temperature and Doppler velocity.

\section{Results}

The activity of ${ }^{125 \mathrm{~m}} \mathrm{Te}$ in the BeTe rods was determined by measuring the gammaray count rate with the intrinsic germanium detector. The gamma ray intensity was corrected for self-attenuation using the expression,

$$
I=I_{0} \frac{1-e^{-\mu_{0} L}}{\mu_{0} L} \text {, }
$$


where $\mu_{\mathrm{o}}$ is the attenuation coefficient and $L$ is the length of the rod. The measurement was made at a $25-\mathrm{cm}$ distance between the source and the detector. The data and parameters used to convert to activity from count rate are listed in Table 1 . The thermal neutron cross section for production of the ${ }^{125 \mathrm{~m}} \mathrm{Te}$ isomer is also listed in Table 1 and is in good agreement with the results of Alpatov et al. [14]. This value, 0.65 barns, is much larger than the value carried in the CSIRS data base, 0.040 barns [15]. A similar sample of BeTe made with natural tellurium was activated at the same time as the separated isotope. The cross section for the production of the isomer in this sample was determined to be 0.70 barns.

Table $1 .{ }^{125 \mathrm{~m}}$ Te activity and parameters used for cross section determinations.

\begin{tabular}{|l|l|l|l|}
\hline Parameter & Value & Uncertainty & Unit \\
\hline $109 \mathrm{keV}$ Count Rate & $5.26 \times 10^{5}$ & \pm 2 & $\mathrm{cps}$ \\
\hline Detector efficiency & $1.77 \times 10^{-3}$ & $\pm 0.02 \times 10^{-3}$ & \\
\hline Attenuation Coeff. & 4.85 & \pm 0.05 & $\mathrm{~cm}^{-1}$ \\
\hline Length & 0.45 & \pm 0.03 & $\mathrm{~cm}$ \\
\hline Decay Rate gamma & $7.3 \times 10^{6}$ & $\pm 0.7 \times 10^{6}$ & $\gamma \mathrm{ps}$ \\
\hline Conversion Coeff. & 354 & \pm 8 & $\mathrm{e} / \gamma$ \\
\hline Activity & $2.6 \times 10^{9}$ & $\pm 0.3 \times 10^{9}$ & $\mathrm{~Bq}$ \\
\hline Isomer density & $3.0 \times 10^{18}$ & $\pm 0.3 \times 10^{18}$ & Atoms $/ \mathrm{cm}^{2}$ \\
\hline $\begin{array}{l}\text { Neutron capture } \\
\text { cross section }\end{array}$ & 0.65 & \pm 0.07 & $\mathrm{barns}$ \\
\hline
\end{tabular}

The gamma spectra were measured as a function of source to detector distance, temperature and angle. The spectra were taken with and without pile up rejection. It was expected that gamma ray pairs form stimulated emission would be collinear. Therefore, stimulated emission should decrease as one over the source to detector distance squared while the rate of accidental coincident detection of gamma rays would decrease as one over the fourth power of the source to detector distance. The intensity of stimulated emission should also be greater along the longitudinal axis of the beryllium telluride rod. The data are shown in Figures 2 through 5 represented as the ratio of the rate of summed gamma 
rays to the square of the single rate. For accidental coincidences this ratio is the pulse-pair resolving time of the counting system and should be constant over the varied parameters.

The data points in the figures are shown with error bars representing one standard deviation counting statistics. The data in Figures 2 and 3 were obtained without use of pile up rejection logic. The scatter in these data is significantly larger than expected on the basis of counting statistics alone. The increased error was traced to difficulty in subtracting nearby peaks from the activation of the other isotopes of tellurium. Iodine-131 from neutron capture on tellurium-130 produces a Compton edge under the $218.56-\mathrm{keV}$ sum peak that decays with time. Tellurium-127 is produced by neutron capture on Tellurium126 and has a gamma ray at $215.07 \mathrm{keV}$. This gamma ray decays with a 109.2-day half life. Tellurium-121 has a gamma ray at $212.22 \mathrm{keV}$ that decays with a 154.7 -day half life. These gamma rays and Compton scattered events lead to a changing background that is imperfectly subtracted, especially when the photo peak resolution is degraded by pulse pile up.

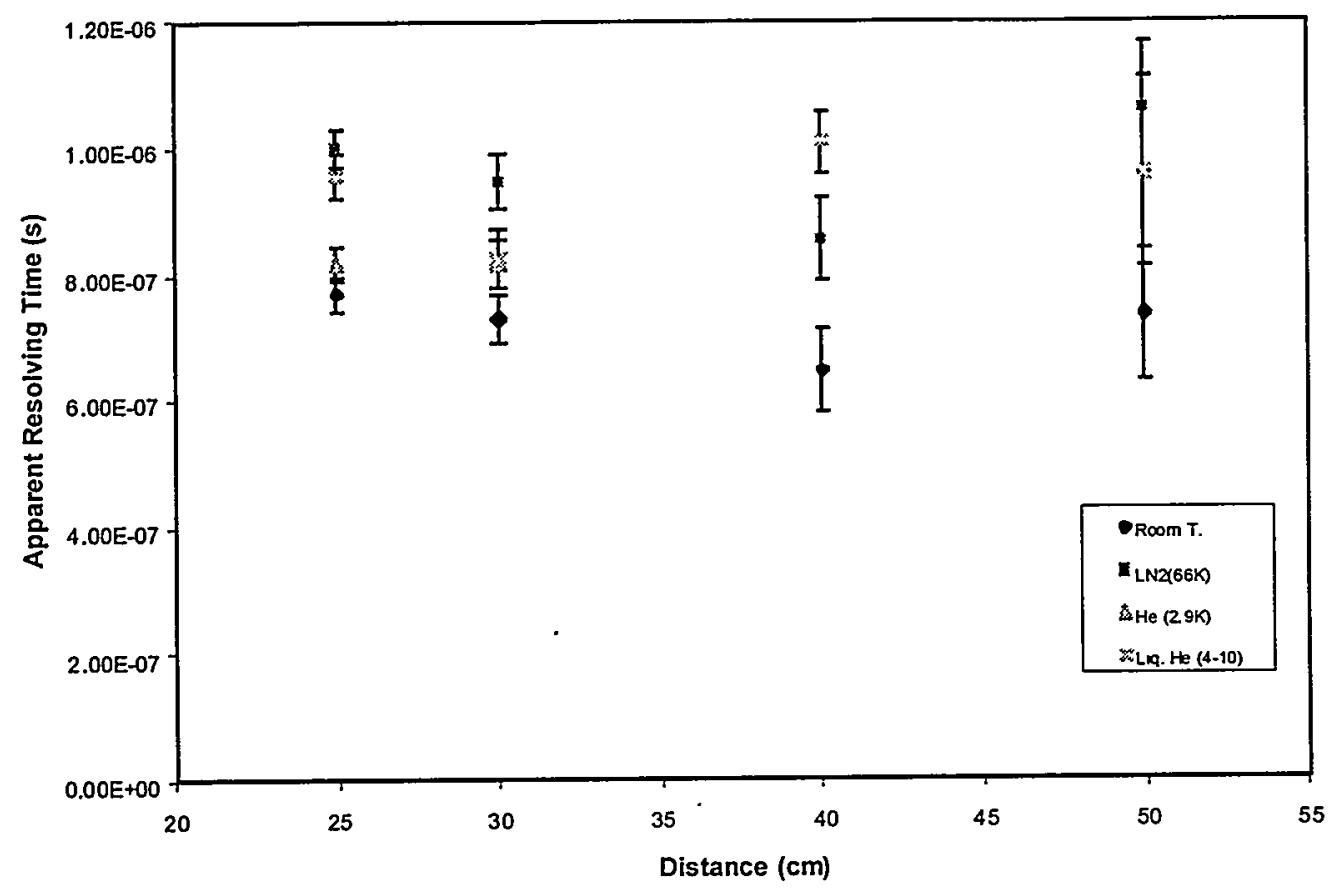

Figure 2. The photo peak summing was measured as a function of sample temperature and source to detector distance. This data set was obtained without pile up rejection logic.

The average of the apparent pulse-pair resolution is $860 \pm 60$ nanoseconds without pile up rejection. This value is large because the shaping time was long at 8 microseconds. The long shaping time was needed to optimize the photo peak resolution at high count 
rates. The width of the sum peak was approximately twice that of the singles peak, about 2 $\mathrm{keV}$. The same ratio for the data shown in Skorobogatov and Dzevitskii [1] would be 740 nanoseconds for the spectrum taken at 10 degrees Kelvin. It is puzzling that the spectrum taken at room temperature shows no sum peak. This implies that the pulse-pair resolving time was less than 80 nanoseconds. This is much shorter than the charge collection time in solid state detectors.

The angular distribution gamma-ray summing is shown in Figure 3. These data are again taken without pulse pile up rejection. The results again show no effect that could be attributed to stimulated emission. The scatter between the data is larger than the counting statistics for the same reasons given above. The singles count rate and sum peak intensities do vary with angle. This variation is solely due the change in attenuation of $109.28-\mathrm{keV}$ gamma rays as the path length through the beryllium telluride rod changes with angle. The minimum count rate is observed at zero degrees. This demonstrates the value of representing the summing of gamma rays as the apparent resolving time. This geometric effect is canceled for accidental coincidences.

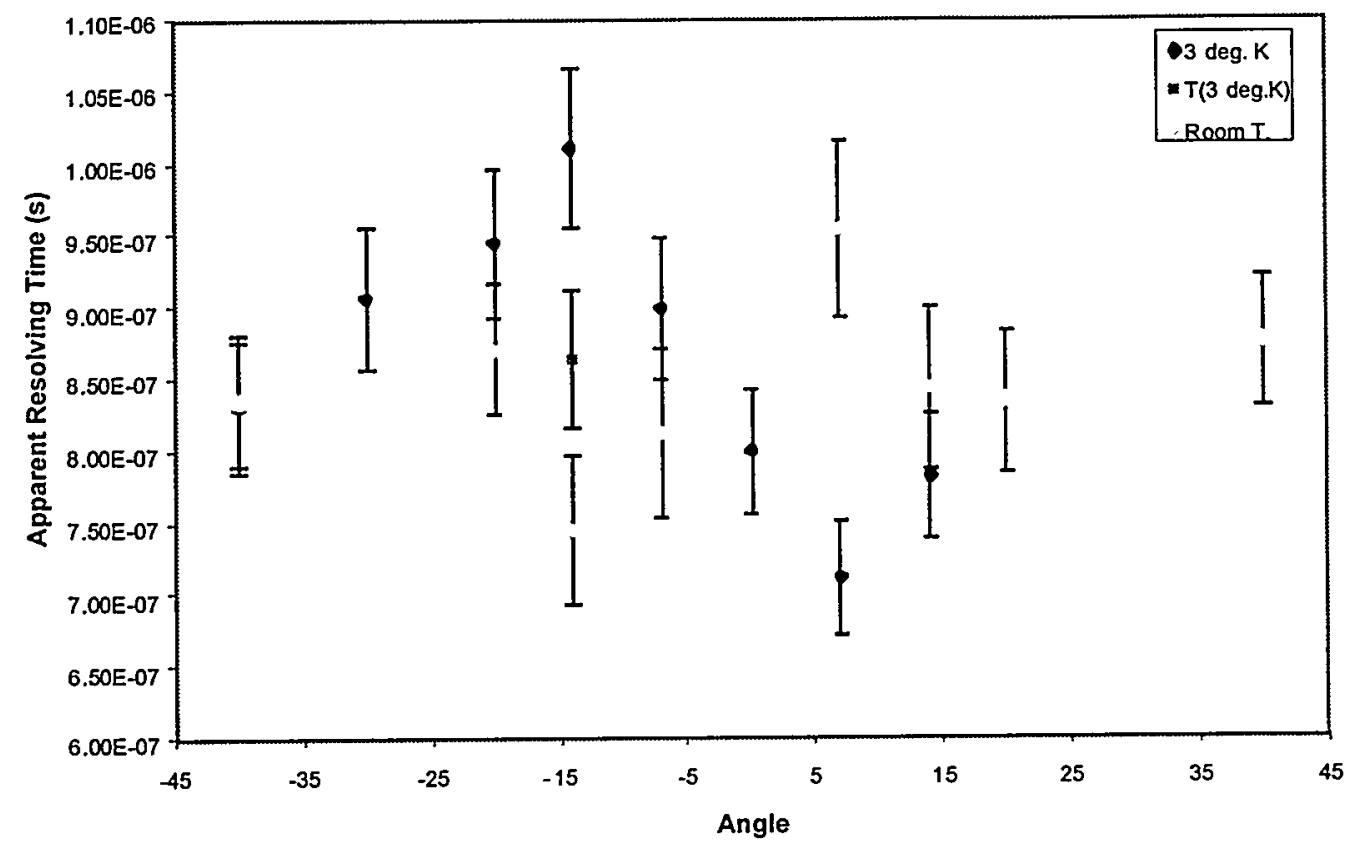

Figure 3. The photo peak summing was measured as a function of sample temperature and angle between the gamma-ray emission direction and the longitudinal axis of the sample. This data set was obtained without pile up rejection logic. 
A second set of data was taken using the linear pulse pile up rejection feature of the Tennelec 245 spectroscopy amplifier. These data are greatly improved. The pulse pair resolving time was reduced to 325 nanoseconds. The scatter of the data conforms to a Gaussian probability distribution with counting statistics. The improvement in the data scatter is due to improve peak resolution making the background subtraction more reliable. The data was taken with and without pulse pile up rejection so that the observation of enhanced gamma ray summing could not be ascribed to an artifact in the handling of dead time due to pulse pile up. The data in Fig. 4 shows no significant increase in the gamma ray summing as the source to detector is increased.

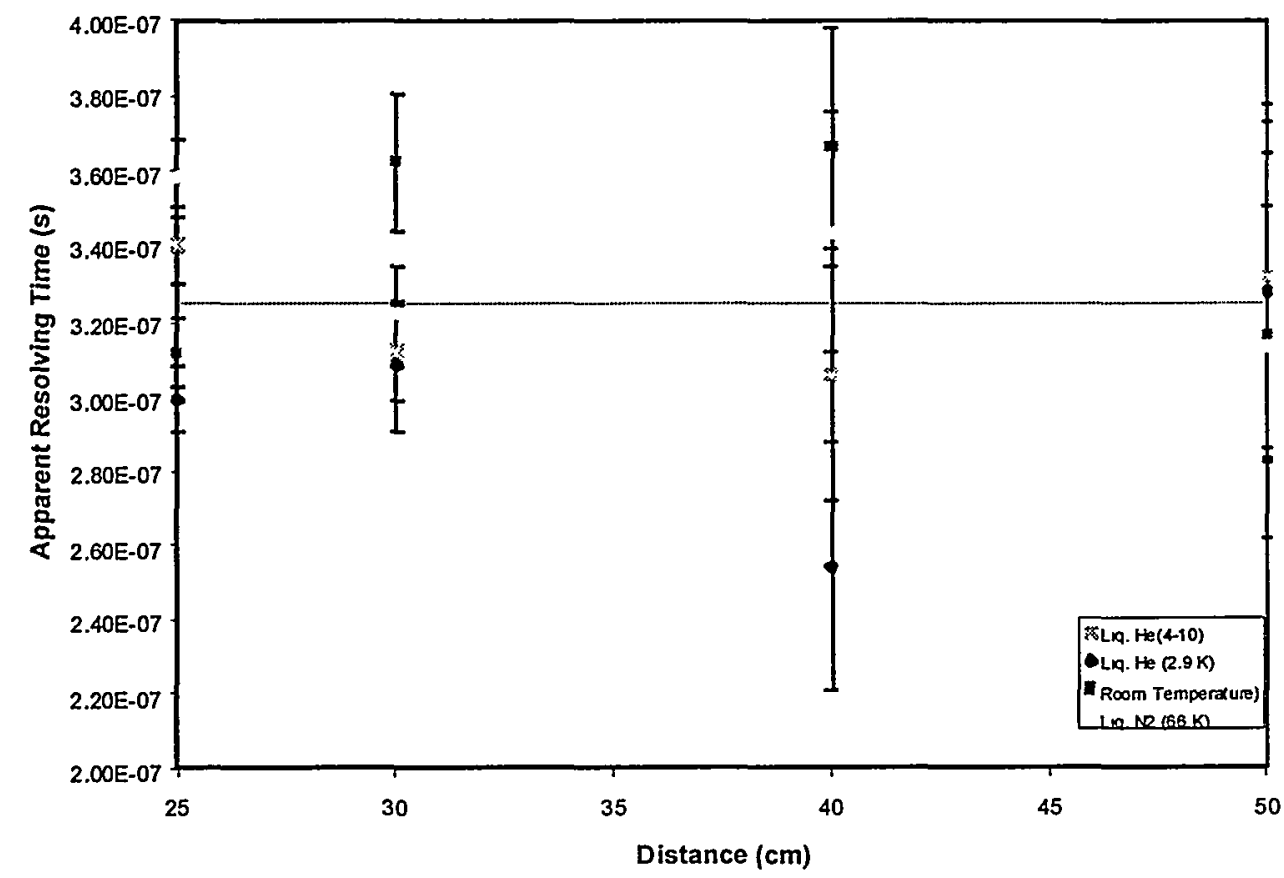

Figure 4. The photo peak summing was measured as a function of sample temperature and source to detector distance. This data set was obtained with pile up rejection logic.

The measurement of summing as a function of angle, shown in Fig.5, again shows no angular dependence that could be characteristic of stimulated emission. The data are in complete agreement with the results shown in Fig. 4.

We were not prepared to make Mössbauer absorption measurements at the time of the first series of measurements. While we had no reason to believe that we had not synthesized pure beryllium telluride, we felt it would be useful to rework the sample with excess 


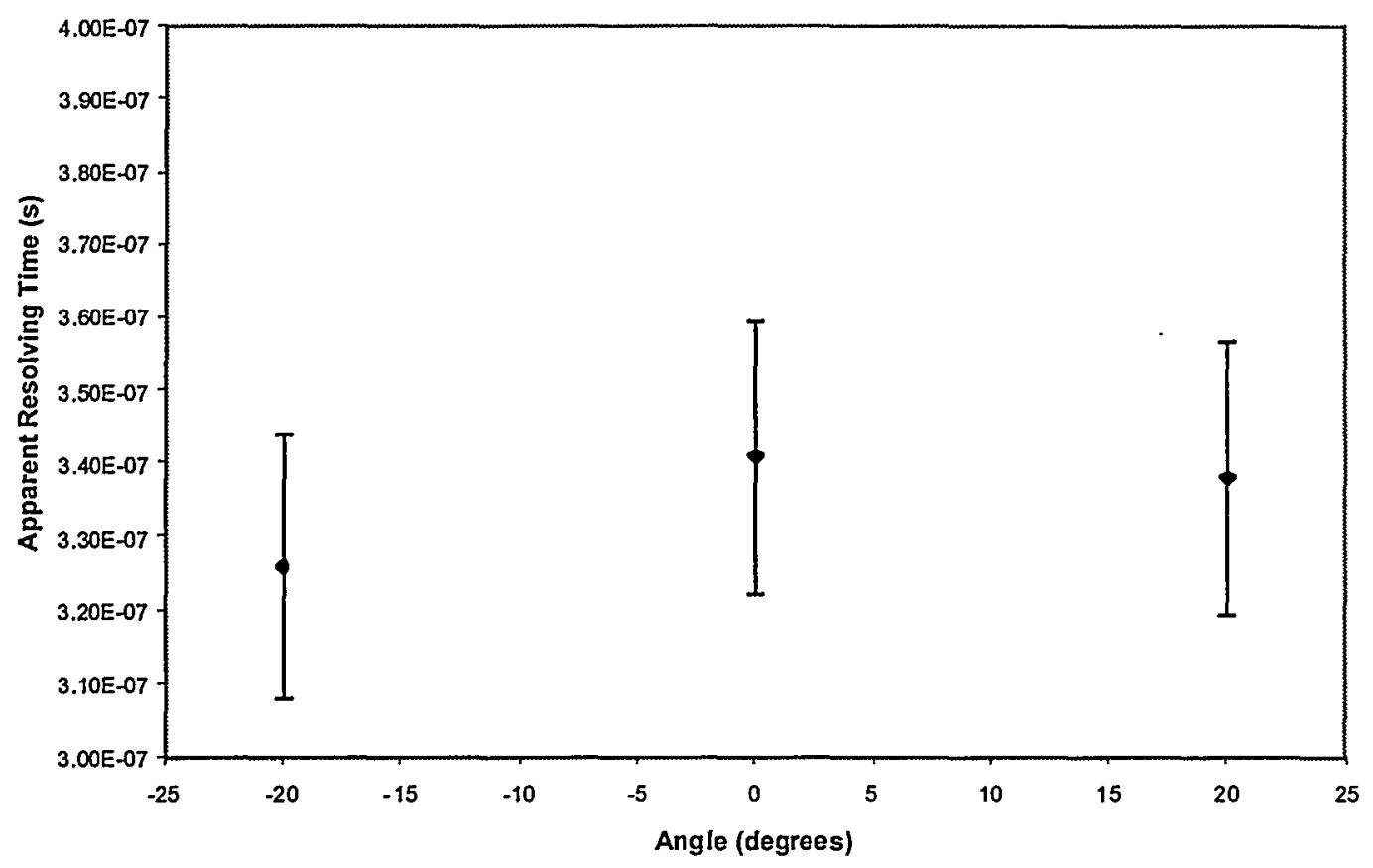

Figure 5. The photo peak summing was measured at liquid helium temperature as a function of angle between the gamma-ray emission direction and the longitudinal axis of the sample. This data set was obtained with pile up rejection logic.

beryllium on the suggestion of Prof. Skorobogatov[16]. Some of the dry runs in the preparation of beryllium telluride prior to the use of separated isotope ${ }^{124} \mathrm{Te}$ had shown evidence of attack on the quartz in spite of the graphite layer. The sample was reacted with excess beryllium using the same procedure as the synthesis of the original sample. The activity in the reworked sample was less than the original because there was some loss in the process and the ${ }^{125 \mathrm{~m}} \mathrm{Te}$ had decayed. The background in the vicinity of the sum peak had also decayed reducing the uncertainty of the later measurements. The data and parameters used to convert to activity from count rate in the reworked sample are listed in Table 2.

The Mössbauer spectrum of the reworked sample was measured using a magnesium tellurate absorber. The recoilless absorption of the $35.5-\mathrm{keV}$ gamma ray was observed even at room temperature. The data were fit to a Lorentzian distribution. The line width was only slightly broader than the natural line width. This indicated that the beryllium telluride 
Table 2. ${ }^{125 \mathrm{~m}} \mathrm{Te}$ activity and parameters used for cross section determinations with the reworked sample (source detector distance $23 \mathrm{~cm}$ ).

\begin{tabular}{|l|l|l|l|}
\hline Parameter & Value & Uncertainty & Unit \\
\hline 109keV Count Rate & $7.33 \times 10^{2}$ & \pm 2 & $\mathrm{cps}$ \\
\hline Detector efficiency & $2.09 \times 10^{-5}$ & $\pm 0.02 \times 10^{-5}$ & \\
\hline Attenuation Coeff. & 4.85 & \pm 0.05 & $\mathrm{~cm}^{-1}$ \\
\hline Length & 0.52 & \pm 0.03 & $\mathrm{~cm}$ \\
\hline Decay Rate gamma & $1.8 \times 10^{6}$ & $\pm 0.7 \times 10^{6}$ & $\gamma \mathrm{ps}$ \\
\hline Conversion Coeff. & 354 & \pm 8 & $\mathrm{e} / \gamma$ \\
\hline Activity & $6.5 \times 10^{8}$ & $\pm 0.6 \times 10^{8}$ & $\mathrm{~Bq}$ \\
\hline Isomer density & $4.2 \times 10^{1 \prime}$ & $\pm 0.4 \times 10^{1 /}$ & Atoms $/ \mathrm{cm}^{3}$ \\
\hline
\end{tabular}

was relatively uniform in composition. The absorption cross section was measured as a function of temperature from 298 degrees Kelvin down to 70 degrees. The temperature dependence was fit to determine the Debye temperature of the solid. The Debye temperature was found to be $380^{\circ} \pm 20^{\circ} \mathrm{K}$.

Twenty spectra, each accumulated for four hours, were taken at room temperature and liquid helium temperature. To correct for drift, each spectrum was manually gainshifted in one-channel increments until the photo peaks from contaminant isotopes matched. Ten four-hour room-temperature runs taken before the liquid helium runs were averaged with ten room-temperature runs after the liquid helium runs. This was done to compensate for the decay of contaminants that contribute to the background under the sum peak. The averaged spectra are shown in Fig. 6. No significant change in the rate of summing was observed in reworked samples as a function of temperature. The difference between sum of counts under the 218.56 peak was less than the statistical uncertainty. Three standard deviations in the sum is 0.015 counts per second. This corresponds to 216 counts in the spectrum shown in Fig. 6 (four-hour count length). The apparent pulse-pair resolution was in agreement with the previous measurements. The cross section for stimulated emission was evaluated using the expression

$$
\sigma_{\mathrm{s}}=\frac{\mathrm{A}_{2 \gamma} / \mathrm{A}_{\gamma}}{\overline{\mathrm{f} \varepsilon_{\mathrm{d}}\left(1-\mathrm{e}^{-\mu \mathrm{L}}\right)(2 \mu)^{-1}\left[{ }^{125 m} \mathrm{Te}\right]}}
$$


where $A_{2 \gamma}$ is the rate of summing, $A_{\gamma}$ the singles rate, and $\varepsilon_{d}$ the detector efficiency.

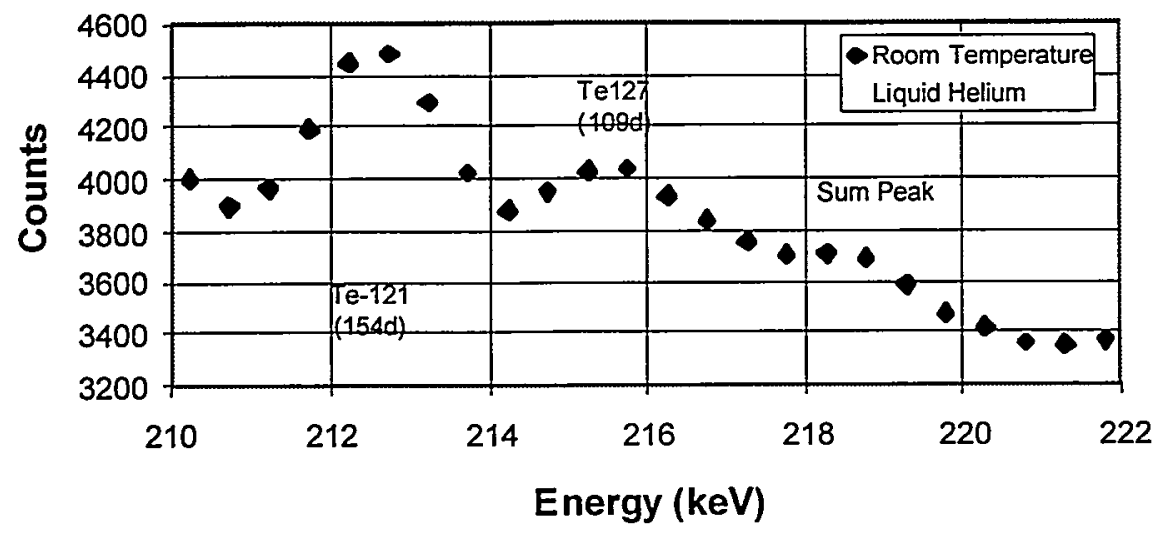

Figure 6. Gamma ray spectra at energy near the $218.56-\mathrm{keV}$ sum peak. The spectra are the average of 20 four-hour spectra.

The detector efficiency was calculated to be 0.76 from calibration standards. The recoilless fraction was 0.10 . Applying the three standard deviation limit gives an upper limit to the cross section for stimulated emission of $6.8 \times 10^{-21} \mathrm{~cm}^{2}$. The first set of data using the original sample gives an upper limit to the cross section of $1.5 \times 10^{-20} \mathrm{~cm}^{2}$ assuming that the Debye temperature is the same as the reworked sample. The limit from the first set is higher simply because the angle and source detector distance was varied so that the total number of counts taken at liquid helium temperature at a single position was much less than for the reworked sample. In addition the Compton edge from ${ }^{131} \mathrm{I}$ had decayed so that the background was less in the reworked sample. 


\section{Conclusions}

The intensity of the sum peak was measured as a function of angle, source-detector distance, and temperature using a coaxial intrinsic germanium detector. The data were represented as the ratio of the sum peak rate to the square of the singles in order to eliminate sensitivity to the reproducibility and precision of the source-detector position as well as the decay of the ${ }^{125 \mathrm{~m}} \mathrm{Te}$. The sum peak ratios observed were all consistent with accidental summing.

No significant difference was observed between the sum peak at room temperature and liquid helium temperature for the reworked sample. The spectra in the vicinity of the sum peak are shown in Fig. 6 . The peaks below the sum peak were identified as activation products from the naturally occurring isotopes of tellurium. The upper limit for the stimulated emission cross section based on the three-standard-deviation statistical error is $6.8 \times 10^{-21} \mathrm{~cm}^{2}$. This result is nearly one order of magnitude lower than the cross section reported by Skorobogatov and Dzevitskii[1]. Our results support the position of Baldwin and Solem [13] over that of Kamenov[11].

The cross section that could be expected in the absence of a mechanism for broadening the photon-channel partial width is $1.5 \times 10^{-22} \mathrm{~cm}^{2}$, based on equation A11 (see Appendix) with an internal conversion coefficient of 352. A cross section of this magnitude could be measured with ${ }^{125 \mathrm{~m}} \mathrm{Te}$ if, for example, a sufficient quantity of ${ }^{125} \mathrm{Sb}$ could be extracted from fission products (i.e., nuclear waste) to manufacture a ${ }^{125 \mathrm{~m}} \mathrm{Te}$ generator. In order for gain to be achieved, either the photon channel would have to be broadened by developing a coherent state in a single crystal, such as has been accomplished in synchrotron radiation experiments [8,9], or the attenuation coefficient reduced via Borrmann channeling[17], or both. These technological advances, while difficult, are not out of the realm of possibility. 


\section{Appendix: Einstein Relations for a Three-Level System}

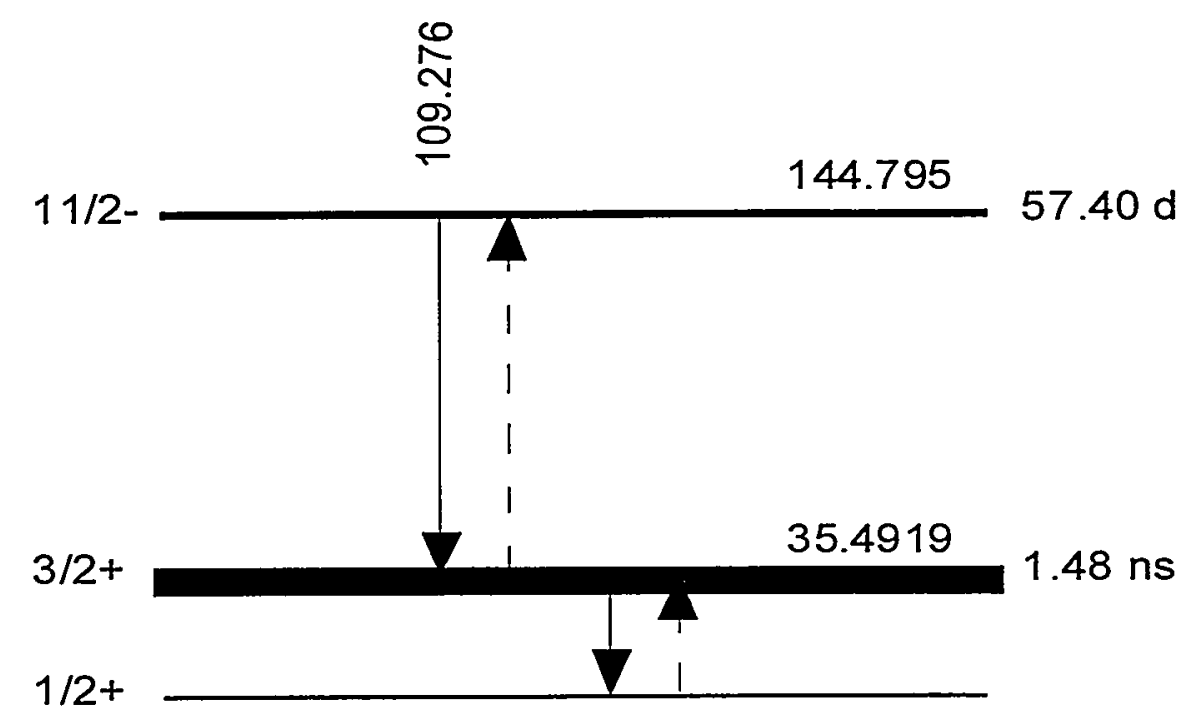

Figure A1. Nuclear levels of ${ }^{125 \mathrm{~m}} \mathrm{Te}$ and reactions in a black body radiation field. Solid arrows represent spontaneous emission and stimulated emission. Dashed arrows represent resonant absorption.

The levels shown in Figure A1 will be referred to as follows: the ground state has the subscript symbol $o$, the first excited state $n$, and the second excited state $m$. The rate that the first excited state is populated in a black body radiation field is,

$$
\mathrm{B}_{\mathrm{on}} \rho\left(v_{\mathrm{no}}\right) \mathrm{W}_{\mathrm{o}}+\left[\mathrm{A}_{\mathrm{mn}}+\mathrm{B}_{\mathrm{mn}} \rho\left(v_{\mathrm{mn}}\right)\right] \mathrm{W}_{\mathrm{m}}
$$

where the Einstein coefficient ${ }^{b_{\text {on }}}$ indicates a photon-induced transition and the subscript refers to the initial and final states respectively (the probability of the ground state being excited to the first excited state by a photon with energy $\left.\urcorner v_{\text {no }}\right), \rho\left(v_{\text {no }}\right)$ is the black body radiation density, and $V V_{O}$ is the Boltzmann population of the ground state. The Einstein coefficient $A_{m n}$ indicates decay and the subscript again refers to the initial and final states, respectively. The rate that the first excited state is depleted can be represented as, 


$$
\left[\mathrm{B}_{\mathrm{no}} \rho\left(v_{\mathrm{no}}\right)+\mathrm{A}_{\mathrm{no}}+\mathrm{B}_{\mathrm{nm}} \rho\left(v_{\mathrm{mn}}\right)\right] \mathrm{W}_{\mathrm{n}}
$$

These rates are equal at equilibrium. The Boltzmann energy distribution is,

$$
W_{i}=g_{i} e^{-\frac{E_{i}}{k T}}
$$

Planck's black-body radiation formula is,

$$
\rho(v)=\frac{8 \pi h v^{3}}{\mathrm{c}^{3}} \frac{1}{\mathrm{e}^{\mathrm{hv/kT}}-1}
$$

Therefore the limit at infinite temperature is,

$$
\lim _{T \rightarrow \infty} \rho(v)=\infty .
$$

Applying this limit one finds,

$$
\mathrm{B}_{\mathrm{on}} \mathrm{g}_{\mathrm{o}}+\mathrm{B}_{\mathrm{mn}} \mathrm{g}_{\mathrm{m}} \frac{v_{\mathrm{mn}}^{2}}{v_{\mathrm{no}}^{2}}=\mathrm{B}_{\mathrm{no}} \mathrm{g}_{\mathrm{n}}+\mathrm{B}_{\mathrm{nm}} \mathrm{g}_{\mathrm{n}} \frac{v_{\mathrm{mn}}^{2}}{v_{\mathrm{no}}^{2}}
$$

The population of the second excited state is treated in the same manner as Einstein's original treatment of a two-level system. The equilibrium rates are,

$$
\mathrm{B}_{\mathrm{mn}} \rho\left(v_{\mathrm{mn}}\right)+\mathrm{A}_{\mathrm{mn}} \mathrm{W}_{\mathrm{m}}=\mathrm{B}_{\mathrm{nm}} \rho\left(v_{\mathrm{mn}}\right) \mathrm{W}_{\mathrm{n}}
$$

Applying the high temperature limit gives,

$$
B_{m n}=\left(\frac{g_{n}}{g_{m}}\right) B_{n m}=A_{m n}\left(\frac{c^{3}}{8 \pi h v_{m n}^{3}}\right)
$$

Substituting equations 2,3 , and 7 into equations 1 and 5 yields, 


$$
B_{n o}=\left(\frac{g_{o}}{g_{n}}\right) B_{o n}=A_{n o}\left(\frac{c^{3}}{8 \pi h v_{n o}^{3}}\right)
$$

The same result would have been obtained if the first excited state and ground state were treated as a two-level system. Thus, the cross section for resonant absorption and stimulated emission are unaffected by the width of levels below. This supports the results and conclusions of Baldwin and Solem [2].

The derivation of formula for the cross section follows the outline in Weinberg and Wigner [18] for the Breit-Wigner formula. The cross section for absorption of photons exciting a nuclear level has a very sharp maximum with a very narrow width compared to the black-body radiation field. The rates of spontaneous decay, stimulated emission, and resonant absorption in the Einstein relations are integrated over the absorption line shape. The other energy-dependent factors are treated independently of the integral because the line width is very narrow. Planck's law is in units of energy density. In order to get the units of the Einstein coefficient $B$ into a rate, the integral cross section must be multiplied by the speed of light. Likewise to get the units of the Einstein coefficient A into a rate of energy transfer, the decay rate must be multiplied by photon energy. Thus equation $A 7$ for stimulated emission becomes ,

$$
c \int \sigma(E) d E=\Lambda h v_{m n}\left(\frac{c^{3}}{8 \pi h v_{m n}^{3}}\right)
$$

where $\Lambda$ is the decay constant. The decay constant is just the level width $l^{\prime} \gamma$ over $n$. Thus the normalization constant for the cross section for stimulated emission becomes,

$$
\int \sigma(E) d E=\frac{\lambda^{2} \Gamma_{\gamma}}{4}
$$

where $\Lambda$ is the wavelength of the photon. If we assume a Lorentzian distribution for the form of the energy dependence, the cross section for the stimulated emission of photons in a three-level system becomes,

$$
\sigma(E)=\frac{\lambda^{2}}{8 \pi} \frac{\Gamma_{\gamma} \Gamma}{(\Gamma / 2)^{2}+\left(E-E_{\circ}\right)^{2}}
$$


This result is identical to the cross section derived for a two-level system (Baldwin, Solem, and Gol'danskii).

\section{References}

1. Skorobogatov, G. A. and Dzevitskii, B. E., Laser Physics 5(2) 258-267 (1995).

2. Mössbauer, R. L., Z. Phys. 151, 124-143 (1958).

3. Terhune, J. H. and Baldwin, G. C., Phys. Rev. Lett. 14(15) 589-591 (1965).

4. Zaretskii, D.F. and Lomonosov, V. V., Soviet Physics JETP 21(1) 243-246 (1965).

5. Hannon, J. P. and Trammel, G.T., Phys. Rev. 186(2) 306-325 (1969).

6. Baldwin, G. C., Solem, J. C. and Gol'danskii, V. I., Rev. Mod. Physics, 53(4) 687744 (1981).

7. Baldwin, G. C., and Solem, J. C., Rev. of Mod. Phys. , V. 69(\#4) 1085-1117 (1997).

8. Chechin, A. I., et al., JETP Lett. 37(11) 633-636 (1983).

9. Faigel, G., et al., Phys. Rev. Lett. 58(25) 2699-2701 (1987).

10. Shyvyd'ko, Y. V., et al., Phys. Rev. Lett. 77(15) 3232-3235 (1996).

11. Kamenov, P.S., Nuovo Cimento D 13(11) 1369-1377 (1991).

12. Einstein, A., and Ehrenfest, P., Z. Phys. 19, 301-306.

13. Baldwin, G. C., and Solem, Nuovo Cimento D 16(6) 627-630 (1994).

14. Alpatov, V. G., et al., Phys. Atomic. Nuclei 58(1) 15-22 (1995).

15. Mughabghab, S. F., Divadeenam, M., and Holden, N. E., Neutron Cross Sections Volume 1, 52-5, Academic Press, New York (1981).

16. Skorobogatov, G. A. (Personal Communication).

17. Borrmann, G., Phys. Z. 42, 157-160 (1941).

18. Weinberg, A. M., and Wigner, E. P., The Physical Theory of Neutron Chain. Reactors, pp. 31-41, The University of Chicago Press, Chicago, (1958). 